\title{
Numerical Solution of Ostrovsky Equation over Variable Topography Passes through Critical Point Using Pseudospectral Method
}

\author{
Nik Nur Amiza Nik Ismail, Azwani Alias*, Fatimah Noor Harun \\ Faculty of Ocean Engineering Technology and Informatics, Universiti Malaysia Terengganu, 21030 Kuala Nerus, Terengganu, Malaysia
}

\begin{abstract}
Cite This Paper in the following Citation Styles
(a): [1] Nik Nur Amiza Nik Ismail, Azwani Alias, Fatimah Noor Harun, "Numerical Solution of Ostrovsky Equation over Variable Topography Passes through Critical Point Using Pseudospectral Method," Mathematics and Statistics, Vol.9, No.5, pp. 825-834, 2021. DOI: 10.13189/ms.2021.090523

(b): Nik Nur Amiza Nik Ismail, Azwani Alias, Fatimah Noor Harun, (2021). Numerical Solution of Ostrovsky Equation over Variable Topography Passes through Critical Point Using Pseudospectral Method. Mathematics and Statistics, 9(5), 825-834. DOI: 10.13189/ms.2021.090523
\end{abstract}

Copyright $@ 2021$ by authors, all rights reserved. Authors agree that this article remains permanently open access under the terms of the Creative Commons Attribution License 4.0 International License

\begin{abstract}
Internal solitary waves have been documented in several parts of the world. This paper intends to look at the effects of the variable topography and rotation on the evolution of the internal waves of depression. Here, the wave is considered to be propagating in a two-layer fluid system with the background topography is assumed to be rapidly and slowly varying. Therefore, the appropriate mathematical model to describe this situation is the variable-coefficient Ostrovsky equation. In particular, the study is interested in the transition of the internal solitary wave of depression when there is a polarity change under the influence of background rotation. The numerical results using the Pseudospectral method show that, over time, the internal solitary wave of elevation transforms into the internal solitary wave of depression as it propagates down a decreasing slope and changes its polarity. However, if the background rotation is considered, the internal solitary waves decompose and form a wave packet and its envelope amplitude decreases slowly due to the decreasing bottom surface. The numerical solutions show that the combination effect of variable topography and rotation when passing through the critical point affected the features and speed of the travelling solitary waves.
\end{abstract}

Keywords Solitary Wave, Nonlinear Equation, Ostrovsky Equation, Variable Topography, Background Rotation, Polarity Change, Pseudospectral Method

\section{Introduction}

The classical model for weakly nonlinear waves propagating over a uniform topography, $h$, yields to the formation of the Korteweg-de Vries (KdV) equation. The effect of the variable topography, however, must be taken into consideration when retrieving the mathematical model. In consequence, the $\mathrm{KdV}$ is succeeded by the variable-coefficient Korteweg-de Vries (vKdV) equation. Johnson [1] was the first person who derive the $\mathrm{vKdV}$ equation for surface waves, in which $Q=c$, and were discussed recently by Grimshaw et al. [2] for internal waves. In the form of the vKdV equation, Grimshaw [3, 4] carried out a detailed analysis and an effective model of solitary wave propagation over the variable topography. On the assumption that the flow is two-dimensional, with $x$ and $z$ indicating horizontal and vertical coordinates, respectively, the end result is

$$
A_{t}+c A_{x}+\frac{c Q_{x}}{2 Q} A+\mu A A_{x}+\lambda A_{x x x}=0,
$$

where $A(x, t)$ is the amplitude of the modal function $\phi(z)$, defined by

$$
\begin{gathered}
\left\{\rho_{0}\left(c-u_{0}\right)^{2} \phi_{z}\right\}_{z}+\rho_{0} N^{2} \phi=0, \text { for }-h<z<0, \\
\phi=0, \text { at } z=-h, \quad\left(c-u_{0}\right)^{2} \phi_{z}=g \phi, \text { at } z=0,
\end{gathered}
$$

that can also be utilized to find the linear phase speed, $c$. Here, $g$ is gravitational acceleration, $\rho_{0}(z)$ is the density of the background that can be defined by the buoyancy frequency $N(z)$ in which, $\rho_{0} N^{2}=-g \rho_{0 z}$ and $u_{0}(z)$ represent the background current, while $t$ is time variables. The coefficient $\mu$ in equation (1) represents the nonlinear term, while $\lambda$ is the coefficient of 
the dispersive term. These coefficients $\mu$ and $\lambda$ are defined by the functionality of the different physical structure where

$$
\begin{aligned}
& I \mu=3 \int_{-h}^{0} \rho_{0}\left(c-u_{0}\right)^{2} \phi_{z}^{3} d z, \\
& I \lambda=\int_{-h}^{0} \rho_{0}\left(c-u_{0}\right)^{2} \phi^{2} d z,
\end{aligned}
$$

and $I$ is written as

$$
I=2 \int_{-h}^{0} \rho_{0}\left(c-u_{0}\right) \phi_{z}^{2} d z
$$

which is obtained by the derivation from the basic equations (see [5] for details). Some theory regarding the solitary waves over the variable topography on the water surface as well as internal solitary waves are discussed [2, 6, 7].

According to $[8,9]$, the $\mathrm{KdV}$ equation is actually augmented with the additional effects known as a background rotation and lead to the formation of the Ostrovsky equation. Several numerical experiments with these equations have shown that the rotational effect inhibits the evolution of internal solitary waves and forms an envelope nonlinear wave packet $[8,10]$. An extension of the $\mathrm{KdV}$ equation which is the Ostrovsky equation (refer Ostrovsky [11] and Grimshaw et al. [12]), is provided by

$$
\left(A_{t}+\mu A A_{x}+\lambda A_{x x x}\right)_{x}=\gamma A,
$$

where the terms inside the bracket are correspond to the $\mathrm{KdV}$ equation, while the coefficient $\gamma$ on the right-hand side represent the rotational effect determined by

$$
I \gamma=f^{2} \int_{-h}^{0} \rho_{0} \Phi \phi_{z} d z,
$$

where $f$ is the Coriolis parameter and

$$
\rho_{0}\left(c-u_{0}\right) \Phi=\rho_{0}\left(c-u_{0}\right) \phi_{z}-\left(\rho_{0} u_{0}\right)_{z} \phi .
$$

The linear dispersion relation of (4) of wavenumber $k$ and frequency $\omega$ for sinusoidal waves, $\sin (k X-\omega t)$, is $\omega=\frac{\gamma}{k}-\lambda k^{3}$. Thus, the phase velocity, $c$ and the group velocity, $c_{g}$ are written as

$$
\begin{aligned}
c & =\frac{\omega}{k}=\frac{\gamma}{k^{2}}-\lambda k^{2}, \\
c_{g} & =\frac{d \omega}{d k}=-\frac{\gamma}{k^{2}}-3 \lambda k^{2} .
\end{aligned}
$$

According to the dispersion relation, the $\mathrm{KdV}$ case $(\gamma=0)$ can support the solitary waves solution for all $c>0$. Meanwhile, the solitary waves solution could not be supported for Ostrovsky equation (4) due to the rotational effect [13]. The situation with $\lambda \gamma>0$, and background current, $u_{0}=0$, that is typical for surface and internal waves, results in unsteady envelope solitary wave solutions [13]. Meanwhile, the circumstances with $\lambda \gamma<0$ which is also described as anomalous case in sufficiently strong shear has been lead to a formation of steady envelope solitary wave $[14,15]$.

Nevertheless, the combination of the variable topography and rotation resulted in the development of the variablecoefficient Ostrovsky equation [16, 17, 18, 19]. These effects is believed can led significant impacts on the propagation of the solitary wave. However, the internal waves usually have a negative polarity which has been proved by the observation of internal wave packets in the deep ocean [20]. This is due to changes in the sign of the coefficient in the quadratic nonlinear term, $\nu$, which is well explained by the KdV theory, [2, 4]. A cubic nonlinear term is sometimes applied to the $\mathrm{KdV}$ equation [2] and to Ostrovsky equation [21, 22] in context of the internal wave with a large amplitude, particularly, when the nonlinearity term, $\mu=0$ is zero. Then the Ostrovsky equation is extended to Gardner Ostrovsky equation

$$
\left(A_{t}+\mu A A_{x}+\mu_{1} A^{2} A_{x}+\lambda A_{x x x}\right)_{x}=\gamma A .
$$

The coefficient $\mu_{1}$ can be found in [23]. In this paper, such cubic nonlinearity is neglected. To conclude, in order to comprehend the Gardner-Ostrovsky equation, one could start by examining the case where the cubic nonlinear term, $\mu_{1}$ is zero and the case when $\mu_{1} \neq 0$ will be a topic for future study. The main interest of this study is to investigate the evolution of the internal solitary wave over variable topography when the nonlinearity term slowly and rapidly passes the critical point in the presence of the background rotation, which is an extension from the studies by $[17,24]$. Similarly, the interest here is still the circumstances where $\lambda \gamma>0$, which is supposed to form an unsteady wave packet.

\section{Problem Formulation}

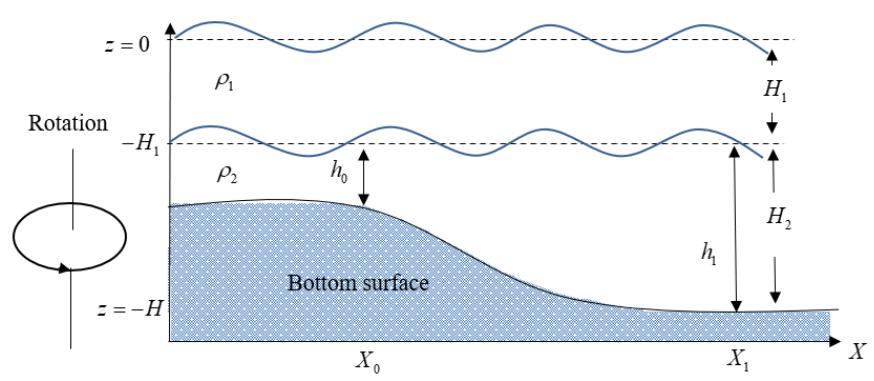

Figure 1. Geometrical configuration of the waves generation over an uneven bottom in two-layer fluid, where $H_{2}>H_{1}$ with rotational effect.

Under the existence of variable topography as the fluid depth, $h$, is slowly changing, the Ostrovsky equation (4) is retrieved by

$$
\left\{A_{t}+c A_{x}+\frac{c Q_{x}}{2 Q} A+\mu A A_{x}+\lambda A_{x x x}\right\}_{x}=\gamma A .
$$

Equation (9) is acknowledged as the variable-coefficient Ostrovsky equation with the additional components corresponding to the slow variability in the basic state. $\mu, \lambda, \gamma, c$, and $Q$ are now in function of $x$ as the modal equation depends parametrically on $x$, where $c(x)$ is the linear long wave speed while $Q(x)$ is the linear magnification factor, so that $Q A^{2}$ becomes the wave action flux for linear long waves given by

$$
Q=I c^{2} \text {. }
$$


Through the transformation

$$
B=\sqrt{Q} A, \quad \tau=\int^{x} \frac{d x}{c}, \quad X=\int^{x} \frac{d x}{c}-t,
$$

equation (9) is now written as

$$
\left\{B_{\tau}+\nu(\tau) B B_{X}+\delta(\tau) B_{X X X}\right\}_{X}=\sigma(\tau) B,
$$

in which

$$
\nu(\tau)=\frac{\mu}{c \sqrt{Q}}, \quad \delta(\tau)=\frac{\lambda}{c^{3}}, \text { and } \sigma(\tau)=\gamma c .
$$

Both coefficients $\nu(\tau)$ and $\delta(\tau)$ are now functions that are defined by the set of the basic state of the fluid, $h=h(\tau)$. Normally, we indicate $A(x, t)=A(X, \tau)$ with $h(x)=h(\tau)$ in which the depth varies gradually along the propagation direction of $x$. Like the $\mathrm{KdV}$ equation, (12) (when $\sigma=0$ and the coefficient is constant) has the initial solitary wave solutions $[25,26]$ that can be written as

$$
B(X, 0)=a \operatorname{sech}^{2}(k X), a=\frac{12 \delta k^{2}}{\nu},
$$

where the speed, $c$ is proportional to the wave amplitude, $a$ and to the square of the wavenumber, $k^{2}$. In defining the internal wave, two-layer fluids are a typical model that can be used. Consider the density in the upper layer, $\rho_{1}$ and lower layer, $\rho_{2}$ are fixed for interfacial waves in a two-layer fluid, say $\rho_{1}=0.001$ and $\rho_{2}=0.002$. The top layer height is $H_{1}$, and $H_{2}=H-H_{1}$ is the lower layer depth, where $H$ is the total depth of fluid (see Figure 1). For typical internal waves, the free boundary is replaced with a rigid boundary, and hence, $\phi(0) \approx 0$ will thus be the upper boundary condition for $\phi(z)$. Assuming $u_{0}(z)=0$ (no background flow) and setting $\rho_{0}=1$, then the expressions are attained

$$
\begin{aligned}
& \phi=\frac{z+H}{H_{2}}, \text { for }-H<z<-H_{1}, \\
& \phi=\frac{-z}{H_{1}}, \text { for }-H_{1}<z<0 .
\end{aligned}
$$

Substitute (15) into (2), (3) and (5), the coefficients $\mu, \lambda$, and $\gamma$ are now

$$
\begin{aligned}
& c=\sqrt{\frac{g^{\prime} H_{1} H_{2}}{H_{1}+H_{2}}}, \quad \mu=\frac{3 c\left(H_{1}-H_{2}\right)}{2 H_{1} H_{2}}, \\
& \lambda=\frac{c H_{1} H_{2}}{6}, \text { and } \gamma=\frac{f^{2}}{2 c},
\end{aligned}
$$

where $g^{\prime}=g\left(\rho_{2}-\rho_{1}\right) / \rho_{1}$ is reduced gravity. Following [19], the numerical solutions of the equation (12) are obtained on the assumption that the initial parameters for $H_{1}$ and $H_{2}$ are 1.5 and 1.0, respectively, with $f=1$. Noted that, the rotational effect reduced the initial wave amplitude in every case. Therefore, to make it more visible, the initial amplitude, $a_{0}=3$ is used for all cases in this study. Hence, a typical plot of linear dispersion relations for phase velocity (6) and group velocity (7) of the proposed model (12), where the coefficients described by (16) are constants is shown in Figure 2.

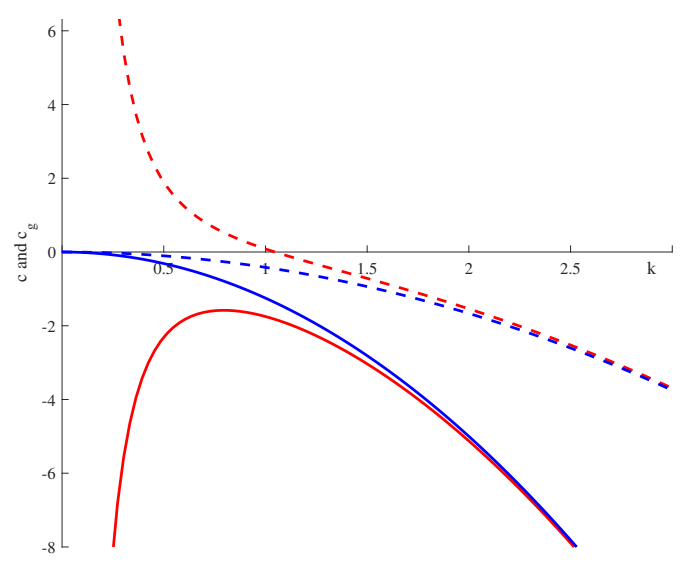

Figure 2. The phase velocity, $c$ (dashed) and group velocity, $c_{g}$ (solid) of equation (12) over a constant depth, $H$, where red is when $\sigma \neq 0$ and blue is when $\sigma=0$.

According to the dispersion relation in the Figure 2, similarly, the gap in $c>0$ for $\sigma=0$ shows that solitary waves can exist with the travelled velocity being positive for all wavenumbers, $k$. On the other hand, no solitary waves exist for all $c$ when $\sigma \neq 0$. The solitary waves are disintegrated here, and the wave packets emerges with group velocity, $c_{g}$ is negative for every $k$. Here, the local maximum group velocity of the problem is $c_{g m}=-1.5811$ at $k_{m}=0.7953$.

In most physical problems, the coefficient $\lambda$ is remains positive for any $X$. However, an interesting condition occurs when the nonlinearity term, $\mu$, passes through a critical point and changes sign at a particular location where $H_{2}>H_{1}$ in the deeper water, resulting in an internal wave of depression. It is believed that, in a non-rotating variable medium, $\sigma=0$, the formation of a solitary wave train is categorized into two situations, according to whether $\mu$ change its sign or otherwise. These situations have been discussed in detail by Grimshaw [27]. Similarly, the depth of the upper layer here is considered constant for all $X$ and the depth of the lower layer varies monotonically from $h_{0}$ to $h_{1}$ (refer to Figure 1 ) in the interval $X_{0} \leq X \leq X_{1}$.

\section{The Pseudospectral Method}

The numerical approach to solve equation (12) is Pseudospectral (PS) method. The numerical solution of the equations is captivating and delicate task, and because of this, only a few numerical studies for Ostrovsky equations have been conducted. This method has been selected among many researches in solving the nonlinear equation especially the integral part when the rotation is added. PS method can considerably speed up the calculation when using Fast Fourier Transform (FFT) which is proven to be a very effective algorithm for computing the Discrete Fourier Transform (DFT). The PS approach uses the Fourier transform to transform spatial derivatives of the PDEs and substitutes the temporal derivative by finite-difference approximation, resulting in a 3-level scheme that needs to be numerically solved [7]. Many numerical codes were generated to solve the constant Ostrovsky equation, see 


\section{$[9,13,15]$.}

The equation (12) is integrated in space $\tau$ by the leapfrog finite-difference framework in the temporal time $X$. Then, the infinite interval is supplemented by $-L<X<L$ with a substantial $L$ to preserve the assumptions of periodicity. Initially, by implementing $\xi=s X+\pi$, the solution interval $[-L, L]$ is transformed into the periodicity interval $[0,2 \pi]$, where $s=\pi / L$, so then $B(X, \tau)$ in (12) is transformed into $U(\xi, \tau)$ as

$$
\left\{U_{\tau}+\nu(\tau) s U U_{\xi}+\delta(\tau) s^{3} U_{\xi \xi \xi}\right\}_{\xi}=\frac{\sigma}{s} U
$$

It is now convenient to use $W(\xi, \tau)=\frac{1}{2} s U^{2}$ notation for the nonlinear terms. Therefore, nonlinear term in (17) is simplified to

$$
\left\{U_{\tau}+\nu(\tau) W_{\xi}+\delta(\tau) s^{3} U_{\xi \xi \xi}\right\}_{\xi}=\frac{\sigma}{s} U
$$

To obtain the numerical solution of $(18),[0,2 \pi]$ interval is then discretised by $N+1$ equidistant points. Let $\xi_{0}=$ $0, \xi_{1}, \xi_{2}, \cdots, \xi_{N}=2 \pi$, so that $\Delta \xi=\frac{2 \pi}{N}$. In the following, we assume $N$ is power of two. By letting $m=\frac{N}{2}$, the DFT of $U\left(\xi_{j}, \tau\right)$ for $j=0,1,2, \cdots, N-1$, written as $\widehat{U}(p, \tau)$, is now provided by

$$
\widehat{U}(p, \tau)=F(\widehat{U})=\frac{1}{\sqrt{N}} \sum_{j=0}^{N-1} U\left(\xi_{j}, \tau\right) e^{-\left(\frac{2 \pi j p}{N}\right) i}
$$

where $p=-m,-m+1,-m+2, \cdots, m-1$ while $i=\sqrt{-1}$, the typical imaginary number. The inverse Fourier transform of $\widehat{U}(p, \tau)$ for $p=-m,-m+1,-m+2, \cdots, m-1$, indicated by $U\left(X_{j}, \tau\right)$ now expressed by

$$
U\left(X_{j}, \tau\right)=F^{-1}(\widehat{U})=\frac{1}{\sqrt{N}} \sum_{p=-m}^{m-1} \widehat{U}(p, \tau) e^{-\left(\frac{2 \pi j p}{N}\right) i}
$$

where $j=0,1,2, \cdots, N-1$, while $F(\widehat{U})$ and $F^{-1}(\widehat{U})$ are DFT and inverse Fourier transform respectively. The given transformations (19) and its inverse, (20) can be accomplished efficiently using the FFT algorithm, in which greatly minimizes the total number of calculations needed. The derivatives of $U$ with respect to $X$ can be determined using

$$
\frac{\partial^{n} U}{\partial X^{n}}=F^{-1}\left\{(i p)^{n} F\{U\}\right\}, \quad n=1,2, \cdots .
$$

Then, the DFT with respect to $X$ of (18) provides

$$
\widehat{U}_{\tau}+i \nu(\tau) p \widehat{W}-i \delta(\tau)(s p)^{3} \widehat{U}=-\frac{i \sigma}{p s} \widehat{U}
$$

The hat represents the Fourier transform. The approximations listed below can be used

$$
\begin{aligned}
\widehat{U}_{\tau} & \approx \frac{\widehat{U}(p, \tau+\Delta \tau)-\widehat{U}(p, \tau-\Delta \tau)}{2 \Delta \tau}=\frac{\widehat{U}^{k+1}-\widehat{U}^{k-1}}{2 \Delta \tau} \\
\widehat{U} & \approx \frac{\widehat{U}(p, \tau+\Delta \tau)-\widehat{U}(p, \tau-\Delta \tau)}{2}=\frac{\widehat{U}^{k+1}+\widehat{U}^{k-1}}{2}
\end{aligned}
$$

Then, substitute (23) into (22), the forward scheme for (12) is attained in the following format

$$
\widehat{U}^{k+1}=\frac{\widehat{U}^{k-1}\left[1+i\left[\Delta \tau \delta(\tau)(s p)^{3}-\frac{\sigma}{p s} \Delta \tau\right]\right]-2 i \Delta \tau \nu(\tau) p \widehat{W}}{1-i\left[\Delta \tau \delta(\tau)(s p)^{3}-\frac{i \sigma}{p s} \Delta \tau\right]}
$$

Equation (24) is a three-level scheme, where to get the third level, $\widehat{U}^{k+1}$, one needs to identify the first level, which is the initial condition that shall subsequently refer it as $\widehat{U}^{k-1}$ and subsequent second level, $\widehat{U}^{k}$. The step is redo until the required $\widehat{U}^{k+1}$ is achieved. In obtaining the second level, $\widehat{U}^{k}$, the interval between $\widehat{U}^{k-1}$ and $\widehat{U}^{k}$ is divided into ten sub intervals. Hence, $\Delta \tau$ in (24) is substituted by $\frac{\Delta \tau}{10}$ to obtain the equation for $\widehat{U}^{k}$ as

$$
\widehat{U}^{k}=\frac{\widehat{U}^{k-1}\left[1+i\left[\frac{\Delta \tau}{10} \delta(\tau)(s p)^{3}-\frac{\sigma}{p s} \frac{\Delta \tau}{10}\right]\right]-2 i \frac{\Delta \tau}{10} \nu(\tau) p \widehat{W}}{1-i\left[\frac{\Delta \tau}{10} \delta(\tau)(s p)^{3}-\frac{i \sigma}{p s} \frac{\Delta \tau}{10}\right]} .
$$

Since the interval between $\widehat{U}^{k-1}$ and $\widehat{U}^{k}$ is divided by ten sub intervals, (25) is evaluated ten times to get $\widehat{U}^{k}$. The values of $L$ and $N$, as well as the required time step $\Delta \tau$ is determined accordingly. The numerical resolution for this problem is $\tau=0.001$ and $N=13684$ on the large time scale to avoid any possible issues. Note that the current simulations are just for the research framework. Table 1 shows the comparison of the solitary wave solution obtained by using (14) and the PS method where the effect of the variable topography is omitted.

\section{Results and Discussions}

The effect of nonlinearity term on the formation of wave in the presence and absence of background rotation is shown in the next Subsection 4.1 and 4.2. The initial KdV solitary wave solution in (14) is used as initial condition:

$$
B(X, 0)=3 \operatorname{sech}^{2}(k X), \quad k=\sqrt{\frac{\nu}{4 \delta}} .
$$

Generally, a common initial condition for the $\mathrm{KdV}$ equation will form a train of solitary waves, but under the background rotation, this wave is diminished and supplanted by an envelope wave packet. According to [9, 13], the initial solitary wave decays due to rotation by forming inertia-gravity waves and is absolutely emitted on a time scale of

$$
\tau_{e}=\frac{1}{\sigma}\left\{\frac{a_{0} \nu}{12 \delta}\right\}^{1 / 2} .
$$

Based on (27), the extinction time for our problem here is $\tau_{e}=0.4821$, hence, by $\tau \approx 1$, the solitary waves is said to be completely extinguished and formed a wave packet. Under the influence of variable topography, it should be noted that the coefficient $\nu$ in (16) will change its sign if $H_{2}>H_{1}$. The numerical results are categorized into two, i.e., one that the nonlinearity term changed its sign in Subsection 4.2 and one that the nonlinearity term did not change its sign in Subsection 4.1. The case when there are polarity changes is separated into two cases where the depth varies slowly and rapidly. 
Table 1. Numerically determined speed, $c$ and amplitude, $a$ of solitary wave propagating over a constant depth, $H$ where, $\triangle c$ and $\triangle a$ are the absolute error of speed and amplitude respectively.

\begin{tabular}{cccccc}
\hline$\tau$ & $X$ & $a$ & $c=\frac{X}{\tau}$ & $\triangle c$ & $\triangle a$ \\
\hline 10 & 4.02221680 & 2.99999900 & 0.40222168 & $5.1 \times 10^{-4}$ & $1.0 \times 10^{-6}$ \\
20 & 8.03833008 & 2.99999000 & 0.40191650 & $2.0 \times 10^{-4}$ & $1.0 \times 10^{-5}$ \\
30 & 12.05444336 & 2.99997600 & 0.40181478 & $1.0 \times 10^{-4}$ & $2.4 \times 10^{-5}$ \\
40 & 16.07055664 & 2.99997500 & 0.40176392 & $5.0 \times 10^{-5}$ & $2.5 \times 10^{-5}$ \\
50 & 20.09887695 & 2.99999100 & 0.40197754 & $2.6 \times 10^{-4}$ & $9.0 \times 10^{-6}$ \\
60 & 24.11499023 & 2.99997100 & 0.40191650 & $2.0 \times 10^{-4}$ & $2.9 \times 10^{-5}$ \\
70 & 28.13110352 & 2.99998000 & 0.40187291 & $1.6 \times 10^{-4}$ & $2.0 \times 10^{-5}$ \\
80 & 32.14721680 & 3.00000700 & 0.40184021 & $1.3 \times 10^{-4}$ & $7.0 \times 10^{-6}$ \\
90 & 36.16333008 & 3.00000500 & 0.40181478 & $1.0 \times 10^{-4}$ & $5.0 \times 10^{-6}$ \\
100 & 40.17944336 & 3.00003600 & 0.40179443 & $8.0 \times 10^{-5}$ & $3.6 \times 10^{-5}$ \\
\hline
\end{tabular}

\subsection{Transformation of Solitary Wave Without Passes Through Critical Point}

The transformation of the solitary wave when the nonlinearity term, $\nu$ did not change it sign where, $H_{2}<H_{1}$ after the slope is first examined. The cross-section of long time evolution of formation of waves propagating over gradually increasing depth, in the absence and presence of rotational effect is shown in Figure 3. The depth profile of lower layer is given by

$H_{2}(\tau)=\left\{\begin{aligned} 1.0 & : \quad 0 \leq \tau<100 \\ (1+0.00045(\tau-100))^{2} & : \quad 100 \leq \tau<411.50 \\ 1.3 & : \quad \tau \geq 411.50\end{aligned}\right.$

As can bee seen clearly from the Figure 3, the rotation effect, $\sigma$ removed the spectral gap where the solitary wave solution can be occurred and formed the unsteady envelope wave packet. The method used is parallel to the result obtained by [13] which has a good agreement with the theoretical solution and laboratory experiment. The propagation of a smallamplitude trailing shelf behind the solitary wave as it propagates over the deeper region can be observed in Figure 3 (Left), which is in excellent agreement with the result found by $[6,19]$. Here, the number of waves within the envelope is inversely proportional to the wave amplitude where the amplitude of the waves is determined by the greatest amplitude in the envelope [13]. On a much larger temporal scale, the trailing shelf decays into another trailing radiating wave and another wave packets is emerges when the background rotation exists, for example, see Figure 3 (Right) at $\tau=3400$.

However, if the depth continually increases, the amplitude of the waves also decreases, and the waves transform into waves of depression when $\nu$ passes the critical point (where $H_{1}=H_{2}$ ). The wave solutions actually depend on how rapidly $\nu$ changes its sign [3]. It is believed that the solitary wave is transformed into a radiating wavetrain if $\nu$ passes through zero rapidly. If $\nu$ changes slowly, the amplitude of the opposite polarity of trailing develops indefinitely as the solitary wave amplitude reduces. Consider the fact that the number of soliton $N$ is obtained by the ratio $Z=\nu_{+} \delta_{-} / \nu_{-} \delta_{+}$, where if $Z>0$, the polarity does not change, and $N=1+[(\sqrt{8 Z+1}-1) / 2]$. However, when $Z<0$, there is polarity change and no soliton is formed, indicating that the entire solitary wave disintegrates into radiation, according to [6].

\subsection{Transformation of Solitary Wave Passes Through Critical Point}

This section examined the propagation of waves passing the critical points where a dramatic change in the formation of waves is expected to occur. Normally, the solitary wave decays adiabatically and begins to disperse once it propagates over the decreasing slope. However, according to (16), observed that, as $\tau$ climbs, $\nu$ in (12) shifts from positive to negative values when $H_{2}>H_{1}$. The depth profile of the lower layer is taken by

$$
H_{2}(\tau)=\left\{\begin{aligned}
1.0 & : 0 \leq \tau<100 \\
(1+0.0014(\tau-100))^{2} & : \quad 100 \leq \tau<317.02 \\
1.7 & : \quad \tau \geq 317.02 .
\end{aligned}\right.
$$

The adiabatic behaviour breaks when $\nu$ approaches the critical point. When the polarity of the internal solitary wave changes its sign, the leading solitary wave deforms, its amplitude gradually decreases, and an elevation rarefaction wave is produced [24, 28]. At this time, its amplitude decreases while the trailing shelf formed grows in amplitude, see Figure 4 (Left). Based on (29), it is not until $\tau>261$ when $\nu$ start to change its sign and the initial internal solitary wave of the negative trailing shelf (in $H_{2}<H_{1}$ region) becomes an initial disturbance to generate an undular bore, which can also be regarded as a secondary solitary wave $[29,30]$. This formation, also known as a dispersive shock wave, is formed as a result of the steepening caused by the nonlinear effect, which triggered the dispersive effects. This pattern has been observed on the free-surface in Northern Australia and the Andaman Sea, for example, with the leading wave followed by a train of welldeveloped undulations where the leading solitary wave travels at a different velocity than the trailing edge. It happens once the fronts of the nonlinear long waves become very steep. The existence of this phenomenon is due to the reaction between travelling solitary waves and bottom topography, hence being easily disturbed by the change of polarity caused by topography conditions. This undular bore transforms into a linear wave on a large time-scale, due to the diminishing pedestal. Hence, the amplitude of the waves decreases over time.

Figure 4 (Right) shows the complete view of the leading packet structure with different phase and group velocity when propagating under the influence of rotational effect. As can see, the outcome is identical to the case when the coefficients are constant [9], where the initial solitary wave decays and forms 

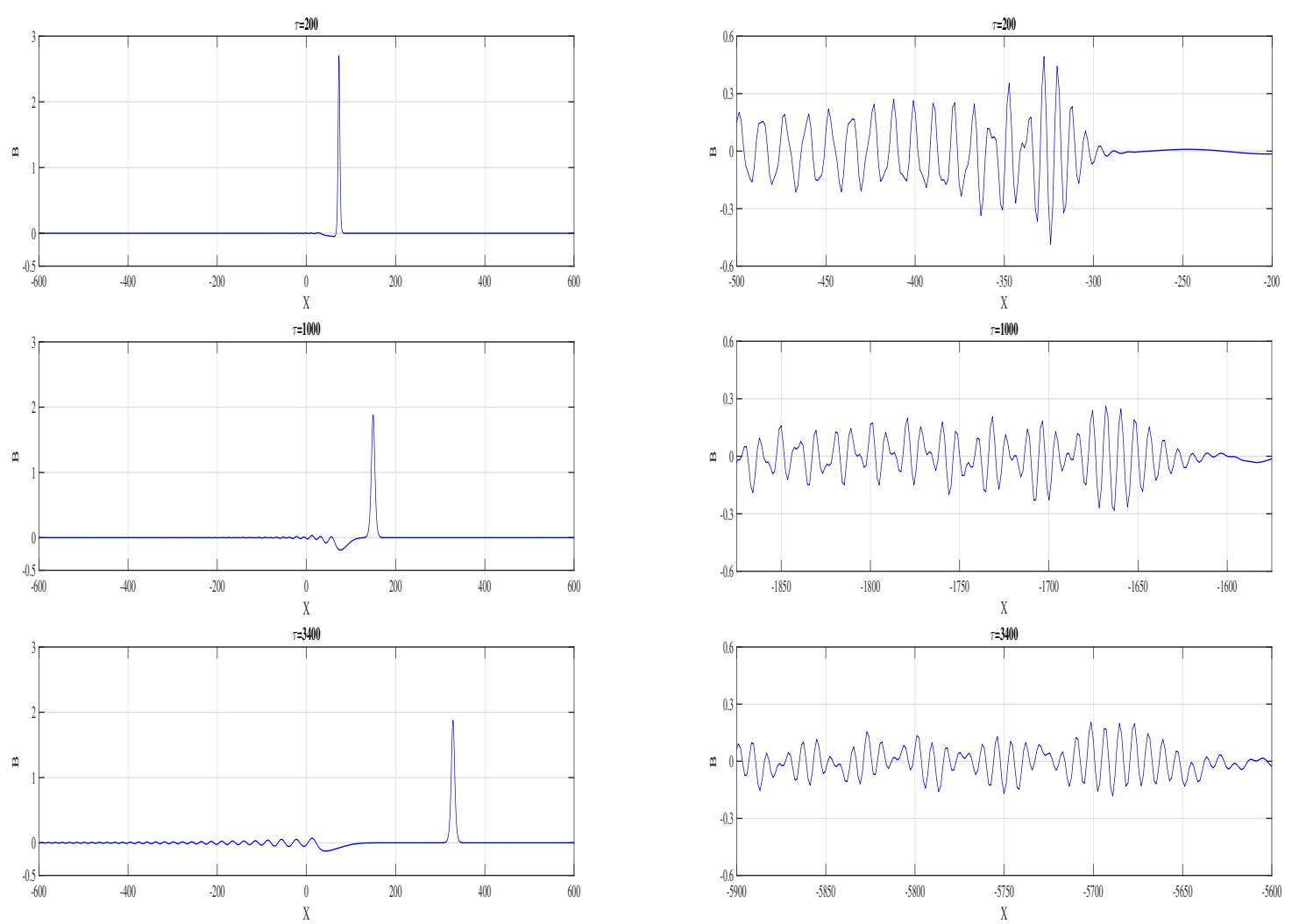

Figure 3. Wave propagation over a slowly increasing depth (28) with the initial condition (14) without polarity change. (Left) Without rotational effect. (Right) With rotational effect.
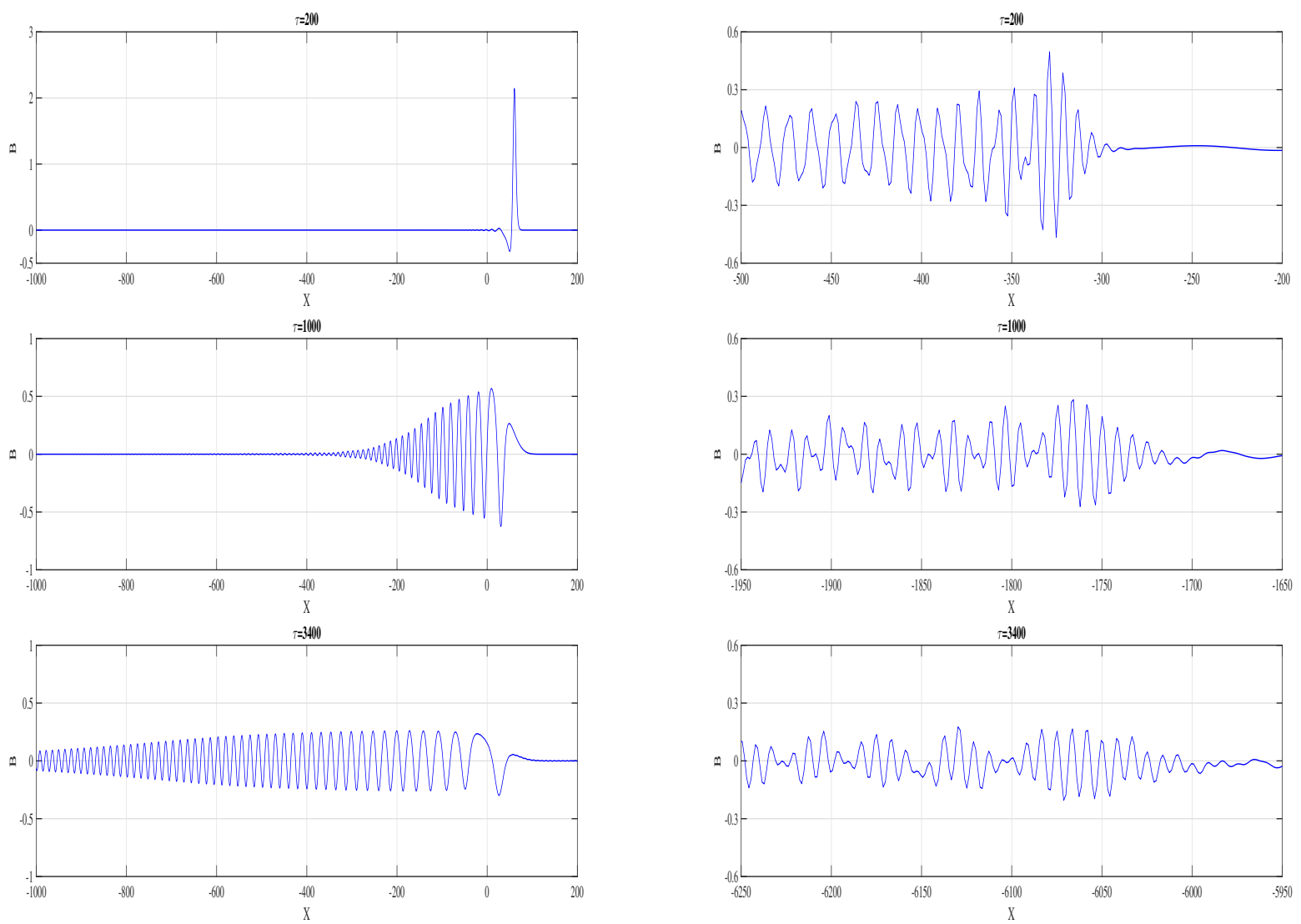

Figure 4. A simulation of the equation (12) when propagating over a slowly increasing depth. The depth profile $H_{2}(\tau)$ is given by (29). (Left) Without rotational effect. (Right) With rotational effect. 
a packet of waves followed by trailing waves. The effect of rotation on internal solitary waves has been observed in the South China Sea [16]. The bigger value of Coriolis frequency or rotational effect chosen, however, has totally extinguished the initial solitary wave and formed a wave packet, a localized disruption caused by the accumulation of numerous different wave forms. The variation in the topography then affects the features and speed of the travelling wave packet. Clearly, the wave packet disperses while propagating due to variable topography. The packet structure with the largest wave amplitude has nonlinear features and satisfied the theoretical solution. Within all the emitted radiation, the wave packet formed has the slowest absolute speed $[8,9]$.

In this case, the effect of rotation tends to be dominant, where the nonlinearity effect is generally suppressed, as the variance of the nonlinear coefficient in the previous case and in this case does not seem to have a significant influence on the subsequent evolution except for the amplitude and speed. It follows that the smaller amplitude of the leading wave and trailing edge of the wave trains lead to the formation of the smaller amplitude of the wave packets and its secondary trailing wave packet, and hence, travelled faster. The numerically obtained speed of nonlinear wave packets (in absolute value) propagating over gradually increasing depth when the nonlinearity term change, (29) and did not change its sign, (28) is given in Table 2 and the comparison is shown in Figure 5.

Table 2. Numerical solution of absolutes speed, $c$ of wave packet propagating over a slowly increasing depth.

\begin{tabular}{cccc}
\hline $\boldsymbol{\tau}$ & $\boldsymbol{H}_{\mathbf{1}}, \boldsymbol{H}_{\mathbf{2}}$ constant & $\boldsymbol{H}_{\mathbf{2}}<\boldsymbol{H}_{\mathbf{1}}$ & $\boldsymbol{H}_{\mathbf{2}}>\boldsymbol{H}_{\mathbf{1}}$ \\
\hline 100 & 1.62963867 & 1.62963867 & 1.62963867 \\
200 & 1.61590576 & 1.63879395 & 1.64489746 \\
300 & 1.62658691 & 1.63370768 & 1.66219076 \\
400 & 1.61209106 & 1.64337158 & 1.70745850 \\
500 & 1.60217285 & 1.64184570 & 1.72485352 \\
600 & 1.60268148 & 1.66117350 & 1.74458822 \\
700 & 1.59781320 & 1.65754046 & 1.74647740 \\
800 & 1.60331726 & 1.66091919 & 1.76315308 \\
900 & 1.59064399 & 1.66219076 & 1.75849067 \\
1000 & 1.59576416 & 1.66320801 & 1.76574707 \\
\hline
\end{tabular}

Next, the wave generation over a rapidly changing slope is examined. The interest is still when there is polarity change, which is when $\nu$ changed its sign rapidly from positive to negative. Both depth profile conditions for $H_{2}$ satisfied $h_{1}>H_{1}$.

$$
H_{2}(\tau)=\left\{\begin{array}{lll}
1.0 & : & \tau<100 \\
1.7 & : & \tau>100
\end{array}\right.
$$

As previously discussed, when the nonlinearity term, $\nu$, rapidly passes through the critical stage, the solitary waves are absorbed and completely transformed into a radiating wave train [3]. Here, the whole structure reduced its amplitude into a radiating wave train. The results obtained shown in Figure 6 (Left) are in agreement with previous work. Although others have been looked at this problem intensively, here we were able to examine further the transformation of a solitary wave at the turning point whenever the background rotation is taken into

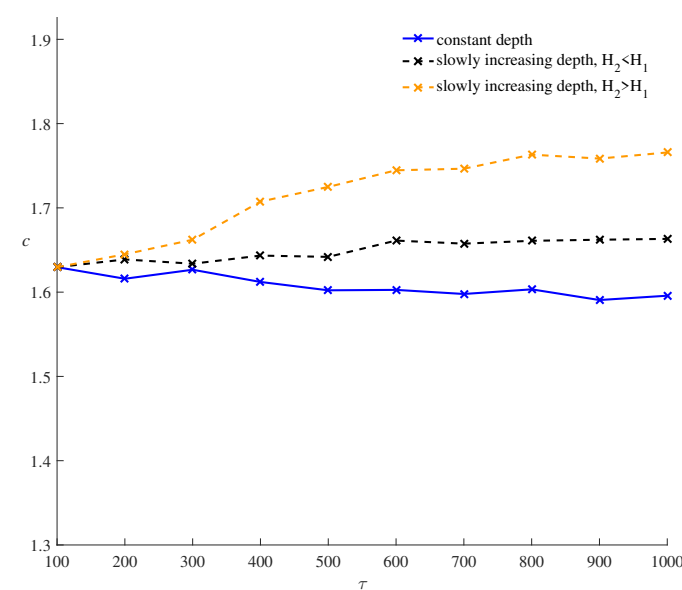

Figure 5. Comparison for the numerically obtained speed, $c$ of the wave packet in slowly changing topography.

consideration. Similarly, the amplitudes of the wave trains, as well as the amplitudes of the wave packets and their trailing radiating waves, decrease over time on a large time scale, as shown in Figure 6 (Right).

These two cases from (29) and (30) involve polarity change in (12) as the waves propagates over the rapidly and slowly increasing depth. When $H_{2}>H_{1}$, a train of solitary waves of negative polarity is formed. Here, as $\sigma=0$, the leading solitary wave has identical amplitude with the trailing shelf close to the critical point $(\nu=0)$, and they are usually smaller than their counterparts without a change in polarity. Likewise, in the case of $\sigma \neq 0$, the combined influence of the nonlinearity and rotation is applied over the solitary wave, and one of the predicted formation outcomes is that if $\nu$ is positive $(\tau \leq 100)$, the result is distinguished by a greater amplitude with a few number of waves in the envelope, as opposed to that in the case of $\nu$ is negative (at $\tau=1000,3400$ ). When the polarity changes, the leading solitary wave train cannot maintain its shape while passing through the critical point and is slowly defeated by the evolving rarefaction waves but the result is distorted due to the combined rotational effect; see the right Figures 4 and 6.

Typically, in this case, the internal waves decay into several wave packets followed by a few residual waves. Any nearly localized packets of waves eventually evolve, each containing a long-wave envelope that is shorter and travelled faster. After a long generation, a different number of wave packets emerge and the formed wave packet becomes more complicated with the presence of more wave trains. The formation of a nonlinear wave packet when $\sigma \neq 0$ depends on the wavetrain formed when $\sigma=0$, as shown in Figures 4 and 6. If the amplitude of the wavetrain is small, then the amplitude of the nonlinear wave packet is also reduced [13]. As a result, the nonlinear wave packet formed will be narrower and the number of the waves inside the envelope wave packet will increase. Furthermore, noticed that the extinction time, (27) for the proposed model is $\tau_{e}=0.4821$, which is too short compared to the total run time in our numerical solution, $\tau \approx 3400$ to take the variation of the nonlinear term into account, resulting in the dominant rotational effect. 

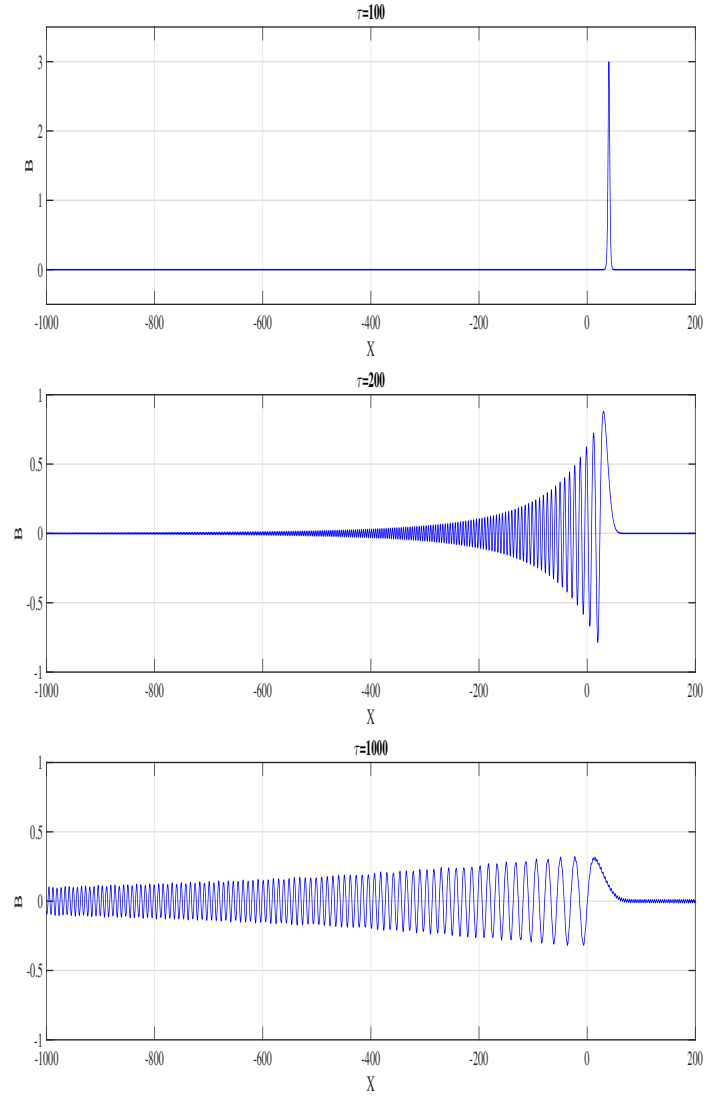
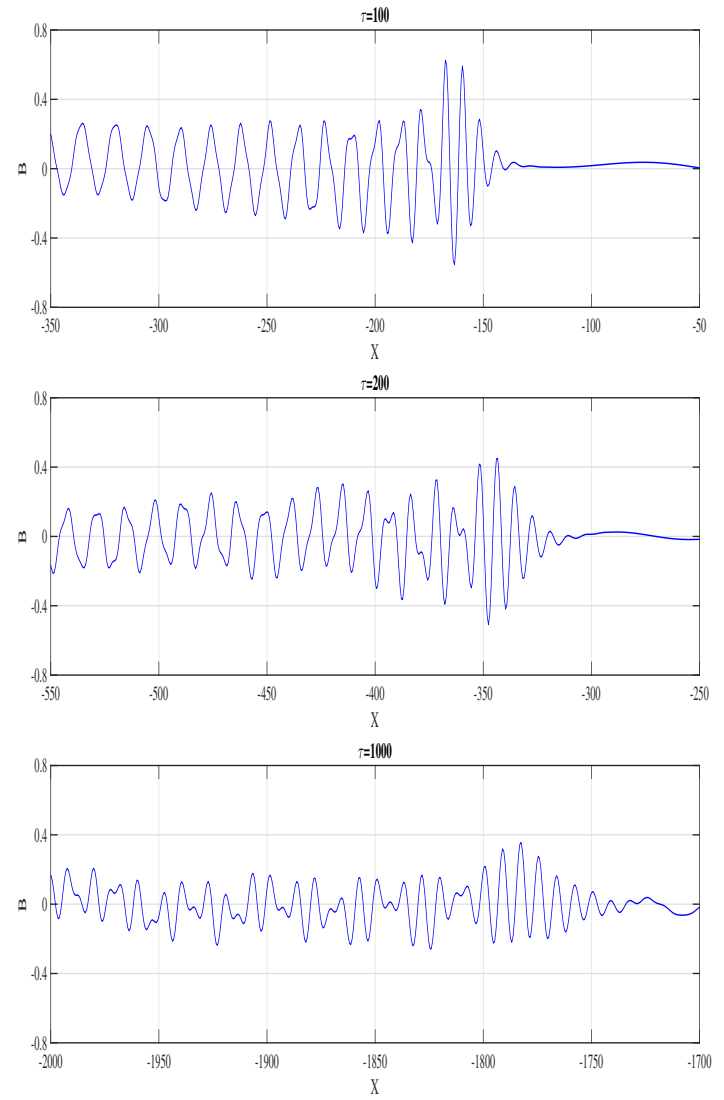

Figure 6. 2D plots for the propagation of KdV-type solitary wave of depression over a rapidly increasing depth (30) where $h_{1}>H_{1}$. (Left) Without rotational effect. (Right) With rotational effect.

\section{Conclusions}

In this study, the formation of an internal KdV-type solitary wave of negative polarity over a rapidly and slowly varying topography in a two-layer fluid system in the presence of background rotation are simulated. The numerical result indicates that the polarity of the internal wave may vary following the depth of the region. If the depth after the slope is greater than the depth of the upper layer, where there is a polarity change, the solitary wave is converted into a train of solitary waves of negative polarity and is accompanied by a radiation wave. At a larger timescale, the amplitude of the solitary waves decreases and diminishes due to the pedestal [24].

However, if there is a background rotation, the wave is gradually replaced by a wave packet accompanied by another secondary trailing wave packet. The existence of secondary trailing wave packet become more obvious after long time evolution. In this situation, the rotational effect seems dominant, with the nonlinear effect indicating some small influence on the evolution of the wave packet which has been supported by [17]. As described, the presented findings could be due to much longer model run-time, as the model runs much longer than the extinction time in (27). Unlike in the KdV case, where nonlinearity has a tremendous impact on the formation of solitary waves, this structure leads to some disruption when the background rotation is applied. As a conclusion, for the case when $\sigma \neq 0$, in both cases (when $\nu>0$ and $\nu<0$ ), the re- sulting speed of the wave packet shows that the wave packets travel faster if the depth is deeper (see Figure 5).

However, the amplitude of the wave packet is still reduced from the initial amplitude, $a_{0}$, due to the rotational effect that makes the energy of the soliton slowly decrease in both cases. In contradiction, the $\mathrm{KdV}$ soliton keeps maintaining its amplitude once it propagates into another constant depth when $\nu>0$, while the leading waves continuously reduce its amplitude when $\nu<0$. Besides, the wave destroys itself when it attempts to pass through the critical point which has been studied numerically by [28]. Nonetheless, the results show that they are consistent with observational studies, that the rotation and nonlinearity terms have a significant impact on the propagation of internal waves [31]. However, these conclusions, of course, must be revised whenever the cubic nonlinear term, $\mu_{1}$ is considered.

As stated earlier, a cubic nonlinear term is frequently applied to the equations, particularly when there is a polarity change. The implications of a solitary wave have been studied thoroughly, which depend on the sign of the cubic term [2,4]. But then, in such an extended KdV equation, a similar theory for the internal waves still has to be developed and therefore would tend to be even more complex. Lastly, it should note that the study has decided to consider the variable-coefficient Ostrovsky equation rather than incorporating other effects to compensate for the loss of the quadratic nonlinear term at the turning point. In fact, the existence of a cubic nonlinear term 
is likely to have a major impact on the process of transformation. The addition of the cubic nonlinear term would almost certainly result in phenomena that are not comparable to those reported here.

\section{Acknowledgements}

The authors would like to thank to anonymous referees for the comments that led to improvements in the paper.

\section{REFERENCES}

[1] Johnson, R. S. "On the Development of a Solitary Wave Moving Over an Uneven Bottom," Mathematical Proceedings of the Cambridge Philosophical Society, Cambridge University Press, 1973, vol. 73, no. 1, pp. 183-203.

[2] Grimshaw, R., Pelinovsky, E., Talipova, T., and Kurkina, O., "Internal Solitary Waves: Propagation, Deformation and Disintegration," Nonlinear Processes in Geophysics, vol. 17, no. 6, pp. $633-649,2010$

[3] Grimshaw, R., "Korteweg-de Vries Equation," in Nonlinear Waves in Fuids: Recent Advances and Modern Applications, Grimshaw, R., Eds., Springer: New York, 2005, pp. 1-28.

[4] Grimshaw, R., "Internal Solitary Waves in a Variable Medium," GAMM-Mitteilungen, vol. 30, no. 1, pp. 96-109, 2006.

[5] Grimshaw, R., Pelinovsky, E., and Talipova, T., "Modelling Internal Solitary Waves in the Coastal Ocean," Surveys in Geophysics, vol. 28, no. 4, pp. 273-298, 2007.

[6] Grimshaw, R., "Solitary Waves Propagating Over Variable Topography," Tsunami and Nonlinear Wave, vol.2, pp. 51-64, 2007.

[7] Nik Ismail, N. N. A. and Alias, A., "Solving Variablecoefficient Korteweg-de Vries Equation Using Pseudospectral Method," ASM Science Journal, vol. 12, no. Special Issue 5, pp 26-33, 2019.

[8] Helfrich, K., "Decay and Return of Internal Solitary Waves with Rotation," Physics of Fluids, vol. 19, no. 2, pp. 026601, 2007.

[9] Grimshaw, R. and Helfrich, K., "Long-time Solutions of the Ostrovsky Equation," Studies in Applied Mathematics, vol. 121, no. 1 , pp. 71-88, 2008.

[10] Grimshaw, R., Silva, J. S., and Magalhaes, J., "Modelling and Observations of Oceanic Nonlinear Internal Wave Packets Affected by the Earth's Rotation," Ocean Modelling, vol. 116, pp. 146-158, 2017.

[11] Ostrovsky, L. A., "Nonlinear Internal Waves in a Rotating Ocean,” Oceanology, vol. 18, pp. 119-125, 1978.

[12] Grimshaw, R. H. J., Ostrovsky, L. A., Shrira, V. I. and Stepanyants, Y. A., "Long Nonlinear Surface and Internal Gravity Waves in a Rotating Ocean", Surveys in Geophysics, vol. 19, no. 4, pp. 289-338, 1998.
[13] Grimshaw, R. and Helfrich, K., "The Effect of Rotation on Internal Solitary Waves," IMA Journal of Applied Mathematics, vol. 77, no. 3, pp. 326-339, 2012.

[14] Whitfield, A. J. and Johnson, E. R., "Whitham Modulation Theory for the Ostrovsky Equation," Proceedings of the Royal Society A: Mathematical, Physical and Engineering Sciences, vol. 473, no. 2197, pp. 20160709, 2017.

[15] Grimshaw, R., Stepanyants, Y., and Alias, A., "Formation of Wave Packets in the Ostrovsky Equation for Both Normal and Anomalous Dispersion," Proceedings of the Royal Society A: Mathematical, Physical and Engineering Science, vol. 472, no. 2185, pp. 20150416, 2016.

[16] Grimshaw, R., Chuncheng, G., Helfrich, K., and Vlasenko, V., "Combined Effect of Rotation and Topography on Shoaling Oceanic Internal Solitary Waves," Journal of Physical Oceanography, vol. 44, no. 4, pp. 1116-1132, 2014.

[17] Chunxin, Y., Grimshaw, R., and Johnson, E., "The Evolution of Internal Undular Bores Over a Slope in the Presence of Rotation," Studies in Applied Mathematics, vol. 140, no. 4, pp. 465-482, 2018.

[18] Stepanyants, Y., "The Effects of Interplay Between the Rotation and Shoaling for a Solitary Wave on Variable Topography," Studies in Applied Mathematics, vol. 142, no. 4, pp. 465-486, 2019.

[19] Nik Ismail, N. N. A., Alias, A. and Harun, F.N., "The Propagation of Nonlinear Internal Waves Under the Influence of Variable Topography and Earth's Rotation in a Two-layer Fluid," Fluids, vol. 5, no. 3, pp. 140, 2020.

[20] Fu, K. H., Wang, Y. H., Lee, C. P., and Lee, I. H., “The Deformation of Shoaling Internal Waves Observed at the Dongsha Atoll in the Northern South China Sea," Coastal Engineering Journal, vol. 58, no. 2, pp. 1650001-1, 2016.

[21] Obregon, M., Raj, N., and Stepanyants, Y., "Adiabatic Decay of Internal Solitons Due to Earth's Rotation within the Framework of the Gardner-Ostrovsky Equation," Chaos: An Interdisciplinary Journal of Nonlinear Science, vol. 28, no. 3, pp. 033106, 2018.

[22] Whitfield, A. J. and Johnson, E. R., "Wave-packet Formation at the Zero-dispersion Point in the Gardner-Ostrovsky Equation," Physical Review E, vol. 91, no. 5, pp. 051201, 2015.

[23] Grimshaw, R., Pelinovsky, E., and Poloukhina, O., "Higherorder Korteweg-de Vries Models for Internal Solitary Waves in a Stratified Shear Flow with a Free Surface," Nonlinear Processes in Geophysics, vol. 9, no. 3/4, pp 221-235, 2002.

[24] Grimshaw, R. and Yuan, C., "The Propagation of Internal Undular Bores Over Variable Topography," Physica D: Nonlinear Phenomena, vol. 333, pp. 200-207, 2016.

[25] Miles, J. W., "The korteweg-de Vries Equation: A Historical Essay," Journal of Fluid Mechanics, vol. 106, pp. 131-147, 1981.

[26] Helfrich, K. R., and Melville, W. K., "Long Nonlinear Internal Waves," Annual Review Fluid Mechanics, vol. 38, pp. 395-425, 2006. 
[27] Grimshaw, R., "Change of Polarity for Periodic Waves in the Variable-coefficient Korteweg-de Vries Equation," Studies in Applied Mathematics, vol 134, no. 3, pp. 363-371, 2015.

[28] Grimshaw, R., Pelinovsky, E., and Talipova, T.,"Solitary Wave Transformation Due to a Change in Polarity," Studies in Applied Mathematics, vol. 101, no. 4, pp. 357-388, 1998.

[29] El, G. A. and Grimshaw, R. H., "Generation of Undular Bores in the Shelves of Slowly-varying Solitary Waves," Chaos: An Interdisciplinary Journal of Nonlinear Science, vol. 12, no.4, pp. 1015-1026, 2002.
[30] Grimshaw, R. H. and Pudjaprasetya, S. R., "Generation of Secondary Solitary Waves in the Variable-coefficient Korteweg-de Vries Equation," Studies in Applied Mathematics, vol. 112, no. 3, pp. 271-279, 2004.

[31] Farmer, D., Li, Q. and Park, J.H., "Internal Wave Observations in the South China Sea: The Role of Rotation and Nonlinearity," Atmosphere-Ocean, vol. 47, no. 7, pp. 267-280, 2009. 